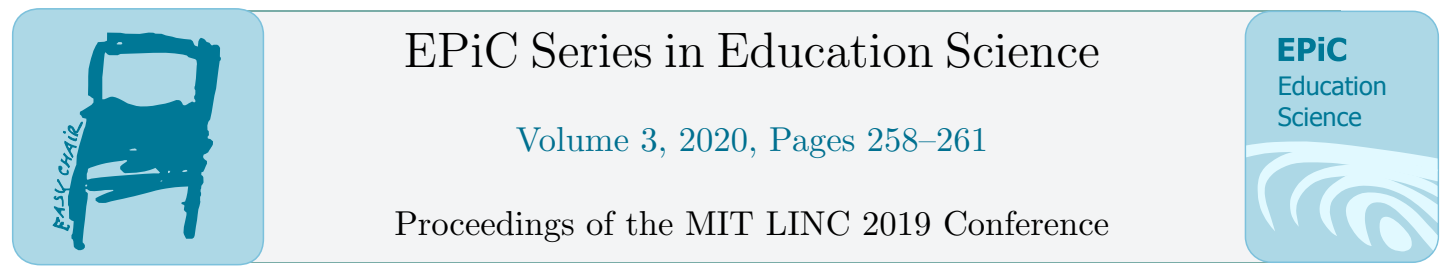

\title{
Embedding the Cartesian Space Principles in a Smile, an Inclusive Learning Method for K12.
}

\author{
Stefano Vuga \\ Eleonora Vuga \\ stefano.vuga@gmail.com \\ eleonora.vuga@pec.it \\ https://cm.alumni.polimi.it/news/stefano-vuga/
}

\begin{abstract}
It is now well established that the negative emotions the child experiences for not understanding a mathematical topic mark their emotional memory associated with that topic. We've been investigating which tangible and accessible tools prevent the development of a pathological allergy to a fundamental concept as it is the Cartesian space, seeking for kid-friendly gates to the subject. When fear and pain for not understanding traces an escape pattern from this topic at a young age the child's ability to relate to all its didactic applications can be seriously jeopardized, marking (when not identified) the school career and sub-sequent attitudes towards all the fields of theoretical and practical application of it.
\end{abstract}

The elementary approach in explaining the Cartesian space principles to the children remains mainly linked to traditional visualization models of three-dimensional images on two-dimensional space, e.g., paper, blackboard, and screens. Only recently, augmented reality has been used as a teaching aid for visualizing objects in the actual three-dimensional space. Those systems are suitable for children naturally predisposed to mathematical and/or visual-cognitive intelligence, who are not suffering from any visual impairment. This is a non-inclusive system of access to understanding such fundamental topic as the Cartesian space. Topic which is later essential to an extended comprehension of geometry, mathematics, representation of objects, and concepts. The aim of the research was to find and test a support system to complement the standard two-dimensional and visual-only approach and to guarantee a complete and consistent sensorial experience of the definition of the Cartesian space through physical, material, and modular forms. We sought to create a bond between the concept and its real representation. This system should be extended to different ages of development and 
types of intelligence and backgrounds, transversal to environments and contexts of usage (family/school), also for visually impaired children. The developed tools pro-vide the child an early and positive emotional bond, prior to any traditional scholastic approach, with the fundamental principles of the Cartesian space through methods such as free play, trial and error, experimentation and share of the emotions while engaging in cooperative activities.

Method // Game and/or assembly actions are completed through a physical system based on the alignment of original blocks (Fig. 1a and 1b) with respect to the three Cartesian axis, identifying in clear and univocal form the three possible directions X, Y and Z. Each block represents one unit. In one direction face-color of the block we advance, and in the opposite face-color we recede, thus with respect to three possible letters-colors. Special shaping of the faces avoids the incorrect assembling of the opponent parts preventing the wrong rotation of the blocks. Further game elements and/or numerical display parts can be attached to/and between the blocks.

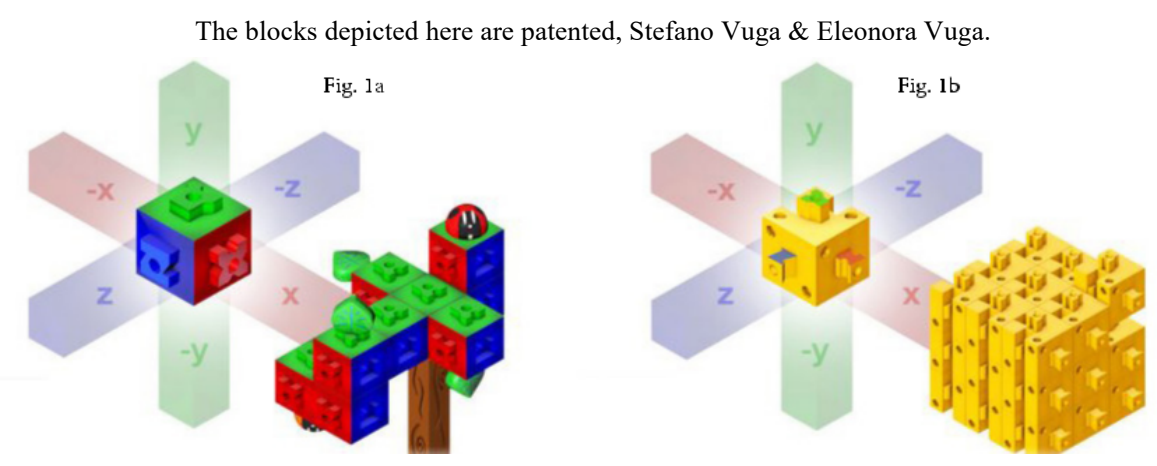

(...) identifying in clear and univocal form the three possible directions ${ }^{2} \mathrm{X}, \mathrm{Y}$ and $\mathrm{Z}$ (Here represented in right hand configuration). Fig.1b, the mono-color construction-block unit differentiates from Fig.1a, a board-game and visualisation tool component, allowing a complete degree of liberty -engaging more with divergent play- while keeping the same axis alignment principle intact.

\section{- Phase 1, free assembly of the parts}

Children are left free to investigate the tools, playing with the two types of blocks as they wish.

- Phase 2, assembly of the parts following game rules (Fig. 2 and Fig. 4)

Children are invited by an adult to play with one of the possible board-game (Fig. 1a) or construction-set (Fig. 1b) application of the system.

- Phase 3, re-assembly of the parts visualizing mathematical concepts (Fig. 3 and Fig. 5)

Using the same blocks, children are invited by the adult to build the three Cartesian axis only and to insert the numerical markers.

\section{- Phase 4, explanation and examples}

Children are invited to listen explanations about the three letter-axis and how to count starting from the centre of the axis representation, using each block as one unit

- Phase 5, verification/repetition of the rules with respect to mathematical concepts (Fig. 5)

Children responds to simple questions about letter/color and (+,-) number direction associations. 


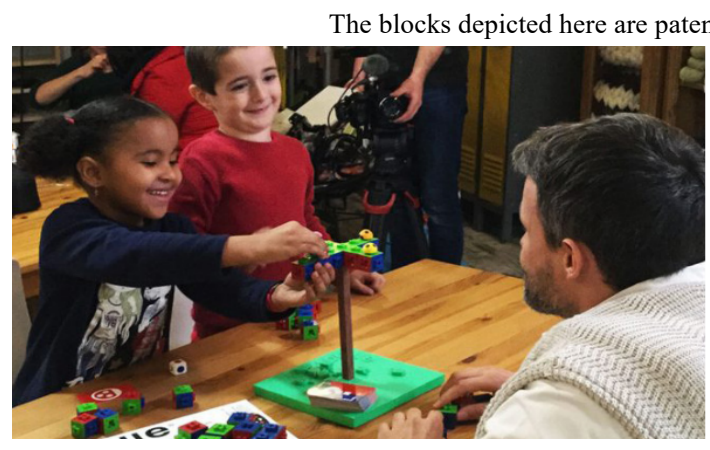

Fig. 4. Phase 2, assembly of the parts following game rules (real footage and tool models) Barcelona, Spain.

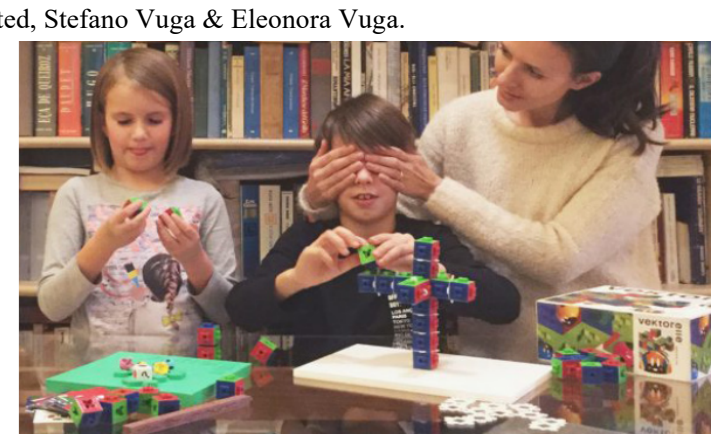

Fig. 5. Phase 3, re-assembly of the parts visualizing mathematical concepts. Phase 5, verification/repetition of the rules with respect to mathematical concepts (real footage and tool models) Bergamo, Italy.

Early results // The first tests on restricted, and heterogeneous, small groups of children (groups of two assisted by an adult) shown how children between the ages of 4 and 8 -first time facing Cartesian space and negative algebraic numbering limited to $+5,-5-$ were able to assimilate these notions in advance compared to the standards defined by the Italian and Spanish ministerial programs.

$\downarrow$ Associate a letter/color with only one direction: Above Below, Left Right, Front Back

$\downarrow$ Define the crossing point of $\mathrm{X}, \mathrm{Y}$ and $\mathrm{Z}$ as the "starting point"

$\downarrow$ Associate a positive order and a negative order "go-forward, go-backward" from the

"starting point" in any of the 3 axis

$\downarrow$ Memorize the above and know how to replicate it without no help following illustrated figures

$\downarrow$ In one case: Understanding and displaying the relativity of the starting point "absolute center" with respect to a starting point different from 0.0.0, a "relative center"

Aid for understanding $\rightarrow$ facilitation of practical understanding $\rightarrow$ sensorial exposure through physical playing. We underline the intent it is not forcing the child in to complex operations. The tool in its essential configuration (Fig. 5) wants to facilitate the correct identification of $\mathrm{X}, \mathrm{Y}$ and $\mathrm{Z}$ or in any case of three fundamental-informations/uniques-references needed to define a point in space, as well the clear visualization of $(+)$ and $(-)$ with respect to 0 . Reinforcing a "good-imprinting" of the basic notions embedded within the games each time the child uses the blocks: Blocks connection = axis orientation (Fig. 1a and 1b) while playing. Further developments of the tool, designed to facilitate algebraicgeometry comprehension and far complex representations are implemented with a consistent system of rigid and flexible sticks, and joints, as well with digital tools.

\section{Early conclusions // - Child side}

- Through the element of play the child is not afraid of experimenting

- If questioned he responds correctly or otherwise tends to approximate to the best possible answer on topics still unknown to his peers, outperforming his peers

- If a topic's solution to a problem is requested through the tool -with respect to the request to write or draw- the child tends to look for solutions and not to flee or distract

- Sharing the act of "building" the elements of the Cartesian space assisted by an educator the kids confronts and reinforce each other in reducing the errors 


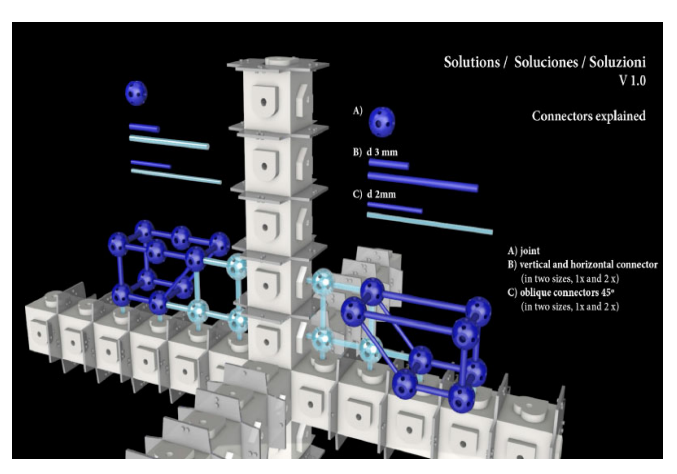

Fig.6. Advanced study for "Giulia". System derived by the commercial product and which will be released under Creative Commons BY by Attribution license in .stl format, authors Stefano Vuga \& Eleonora Vuga (slides). "Giulia" is currently is being optimised with the support of two Teachers of Math for visually impaired kids from the Centro de Recursos Educativos (CRE) de la ONCE, Barcelona.

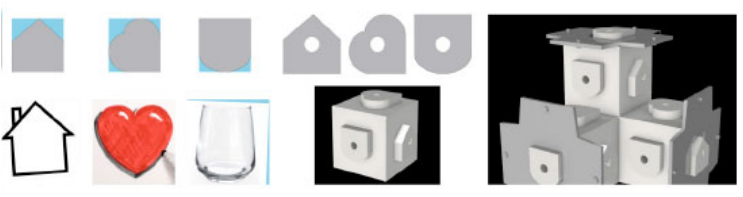

\section{- Educator side}

The child's mental process is broken down through the execution, it becomes transparent spotting potential dynamics of error. The tool helps the educator in identifying at which stage in the acquisition of concepts (axis system and naming, positive and negative numbering, point in the space) the child might experience difficulties. Individual limits and peculiarities of the child in the specific relationship with the topic becomes immediately visible through the actions that they perform.

\section{- Accessibility}

Due to the relevance of the tool's purpose, we are deriving from the patented product a noncommercial (Fig. 6) version tool for visually impaired kids called "Giulia". Released under Creative Commons BY, by attribution license in .stl format and progressively updated. "Giulia" has been optimised with the support of the Teachers of Mathematics for visually impaired kids from the Centro de Recursos Educativos (CRE) de la ONCE, Barcelona.

\section{Bibliography}

Brian Butterworth, Developmental Dyscalculia, 2005

Yvonne Caldera, Children's Play Preferences, Construction Play with Blocks, and Visual-spatial Skills: Are they Related? International Journal of Behavioral Development 23(4):855-872 · December 1999

K. Groos, The Play of Man, New York, Appleton, 1901

Paul Howard-Jones, Jayne Taylor \& Lesley Sutton, The effect of play in the creativity of young children during subsequent activity, Early Child Development and Care, vol 172, 2002

Daniela Lucangeli, É pericoloso rendere patologico ogni errore, CNIS Association for the Coordination of Specialized Teachers and investigation on handicap http://www.cnis.it/en/2016 Shortlink: https://bit.ly/2Wz2jN3 (Italian) Daniela Lucangeli, L'Errore è un sintomo, Università degli Studi di Padova, 2014 http://www.handimatica.com/ Shortlink: https://bit.ly/2WAbe0I (Italian).

Daniela Lucangeli, A. Iannitti, M. Vettore, Lo sviluppo dell'intelligenza numerica, Carocci Editore (Italian), 2007 M.

Montessori, Educare alla libertà, Mondadori (Italian), 2008

Caren M. Walker \& Alison Gopnik, Causality and imagination, University of California, Berkeley

http://alisongopnik.com

\section{Bibliography/Video}

Daniela Lucangeli, Emotional short-circuits: the intelligence behind mistakes, TEDxMilan 2017

https://youtu.be/QuC52IoTczY (Italian, subtitled in English)

D. Lucangeli, Come potenziare l'intelligenza numerica, TEDxCaFoscariU 2014

https://youtu.be/Il4zpiGLxq0 (Italian) 